\title{
Integrating animal movement with habitat suitability for estimating dynamic migratory connectivity
}

\author{
Mariëlle L. van Toor • Bart Kranstauber • Scott H. Newman • Diann J. Prosser • \\ John Y. Takekawa • Georgios Technitis · Robert Weibel • Martin Wikelski • \\ Kamran Safi
}

Received: 31 March 2017 / Accepted: 23 March 2018/Published online: 26 April 2018

(C) This is a U.S. government work and its text is not subject to copyright protection in the United States; however, its text may be subject to foreign copyright protection 2018

\begin{abstract}
Context High-resolution animal movement data are becoming increasingly available, yet having a multitude of empirical trajectories alone does not allow us to easily predict animal movement. To answer ecological and evolutionary questions at a population level, quantitative estimates of a species' potential to link patches or populations are of importance.
\end{abstract}

Electronic supplementary material The online version of this article (https://doi.org/10.1007/s10980-018-0637-9) contains supplementary material, which is available to authorized users.

M. L. van Toor $(\bowtie) \cdot$ M. Wikelski $\cdot$ K. Safi

Department of Migration and Immuno-Ecology, Max

Planck Institute for Ornithology, Am Obstberg 1,

78315 Radolfzell, Germany

e-mail: mlvantoor@gmail.com

M. L. van Toor · M. Wikelski · K. Safi

Department of Biology, University of Konstanz,

Universitätsstrasse 10, 78464 Konstanz, Germany

Present Address:

M. L. van Toor

Department of Biology and Environmental Science, Linnæus

University, Barlastgatan 11, 39182 Kalmar, Sweden

B. Kranstauber

Department of Evolutionary Biology and Environmental

Studies, University of Zurich, Winterthurerstrasse 190,

8057 Zürich, Switzerland
Objectives We introduce an approach that combines movement-informed simulated trajectories with an environment-informed estimate of the trajectories' plausibility to derive connectivity. Using the example of bar-headed geese we estimated migratory connectivity at a landscape level throughout the annual cycle in their native range.

Methods We used tracking data of bar-headed geese to develop a multi-state movement model and to estimate temporally explicit habitat suitability within the species' range. We simulated migratory movements between range fragments, and calculated a measure we called route viability. The results are

\section{S. H. Newman \\ Food and Agriculture Organization of the United Nations, Regional Office for Africa, 2 Gamel Abdul Nasser Road, Accra, Ghana \\ D. J. Prosser \\ U.S. Geological Survey, Patuxent Wildlife Research Center, Beltsville, MD 20705, USA}

\author{
J. Y. Takekawa \\ Suisun Resource Conservation District, 2544 Grizzly \\ Island Road, Suisun City, CA 94585, USA \\ G. Technitis - R. Weibel \\ Department of Geography, University of Zurich, \\ Winterthurerstrasse 190, 8057 Zürich, Switzerland
}


compared to expectations derived from published literature.

Results Simulated migrations matched empirical trajectories in key characteristics such as stopover duration. The viability of the simulated trajectories was similar to that of the empirical trajectories. We found that, overall, the migratory connectivity was higher within the breeding than in wintering areas, corroborating previous findings for this species.

Conclusions We show how empirical tracking data and environmental information can be fused for meaningful predictions of animal movements throughout the year and even outside the spatial range of the available data. Beyond predicting migratory connectivity, our framework will prove useful for modelling ecological processes facilitated by animal movement, such as seed dispersal or disease ecology.

Keywords Anser indicus . Bar-headed goose . Empirical random trajectory generator - Migratory connectivity $\cdot$ Movement model $\cdot$ Stepping-stone migration model

\section{Introduction}

Animal movements and migrations can provide functional connectivity between areas that are separated in geographical space by transporting biomass, genes, and less mobile organisms. This connectivity has wider ecological implications for the species' population structure, and can also provide the dispersal opportunities for organisms like flowering plants by moving pollen and seeds, or pathogens (Altizer et al. 2011; Bauer and Hoye 2014). Identifying connectivity networks and understanding the contribution of animal movement to such networks is a prime motive in ecology and is pivotal to our understanding of spatial structuring processes.

Establishing whether, how, and when animal movement provides a functional connection in space, however, is not easily achieved. Capture-mark-recapture techniques have revealed much about dispersal capabalities of individual animals, thereby providing a history of observed connectivity between distant patches. Estimates such as maximum observed dispersal distances can be used to infer connectivity networks where movement has not been observed, yet there are limitations to their application (Calabrese and Fagan 2004), as distance alone can be insufficient to explain patch connectivity. Estimates of effective distance between patches that incorporate barriers and facilitations to animal movement can be used to improve predictions of connectivity. Algorithms like least-cost paths (e.g., Ferreras 2001; Graham 2001) and electrical circuit theory (McRae et al. 2008) can, in combination with spatially explicit predictors of landscape resistance to movement, provide environmentally informed estimates of connectivity between patches (e.g. for population genetics Row et al. 2010). Often, however, the animal location data used to inform models used for predicting such resistance surfaces lack a behavioural context. Consequently, these resistance surfaces might not be representative of how animals move through the environment (Keeley et al. 2017).

More recently, use of remote tracking technology on wild animals has provided great insights into how, when, and where animals move (Hussey et al. 2015; Kays et al. 2015). Such data are not only a rich source of information about the movement and behaviour of individuals, but can also reveal actual connectivity between spatially separated areas in great detail. In combination with environmental information about the utilised habitat, movement data can provide detailed insight into habitat connectivity for the observed individuals (Almpanidou et al. 2014). Connectivity estimates derived from observed movement, as for example in fragmented landscapes, have been shown to outperform predictions derived from resistance surfaces (LaPoint et al. 2013). Yet to estimate connectivity the use of animal movement data is not without constraints (Calabrese and Fagan 2004). While the miniaturisation of tracking technologies permits scientists to follow ever more individuals of ever smaller species, the cost and effort associated with animal tracking limit sample size, as well as the spatial and temporal extent of the data that can be collected. Thus, the number of individuals that scientists are realistically able to track will remain minuscule compared to even the most conservative estimates of the numbers of moving animals on this planet. The goal of an increasing number of studies is to utilise the knowledge from few, well-studied individuals to estimate the behaviour at a population level. However, such generalisations are not straightforward, mainly because the movement behaviour of individuals and 
the observed variation may not be representative of the population (e.g., Austin et al. 2004). Individual decision-making is not only influenced by general species properties, but also by variation between individuals and their needs and the surrounding environmental conditions (Nathan et al. 2008). Any kind of movement behaviour is thus to some extent unique to the individual, and explicit in time, space, and its environmental conditions as well as its ecological context.

The literature published on models developed for capturing animal movement is extensive, and such models have been shown to provide useful and sensible estimates of the behaviour of observed as well as unobserved individuals (e.g., Morales et al. 2004; Codling et al. 2008; Michelot et al. 2017; Péron et al. 2017). Providing sensible hypotheses of the routes that animals take might require the contextualisation of observed movement, and the understanding of how animals utilise environmental features for route decision-making. Consequently, movement models that incorporate resource-selection functions (step-selection functions, e.g. Fortin et al. 2005; Thurfjell et al. 2014) are becoming increasingly popular. Step-selection functions have been shown to yield functional estimates of how environmental features influence an animal's movement through the landscape (e.g., Richard and Armstrong 2010), and have been used to estimate connectivity between patches (Squires et al. 2013). Such step-selection functions, representing resource selection during actual movement, can be used to derive behaviourspecific predictions for resistance of a landscape to movement. In combination with least-cost paths or circuit theory, these context-aware resistance surfaces provide the means to predict the movement of individuals through the landscape (e.g., Zeller et al. 2014, 2016).

In many cases, animals use series of different movement strategies that change in response to the surrounding environment, or in response to the different needs an animal has for different behaviour or life-history stages. Currently, however, even context-aware approaches used for predicting the movement of unstudied individuals often make the assumption that animals follow a single, constant decision rule. As shown by Zeller et al. (2016), these decision rules are considered to be independent of the supply needs of the individual. We think that realistic movement simulations should not only take the environmental context of movement behaviour into account, but also acknowledge the different movement strategies expressed by a species (see e.g. Morales et al. 2004). One example of such a multi-state movement behaviour with striking differences between states are the stepping-stone-like migrations as performed by many migratory bird species that predominantly use flapping flight for locomotion. Here, we refer to stepping-stone migrations as performed by large waterbirds like ducks and geese covering large distances in fast and non-stop flight and using stopover locations for extended staging periods to replenish their fat reserves. Context-aware, multistate approaches for simulating animal trajectories are uncommon. An additional difficulty for the simulation of stepping-stone migratory movements, is that detailed knowledge about available stopover sites for staging migrants might be necessary.

Here, we introduce a novel approach that allows for inferring environmentally informed migratory trajectories from a multi-state discrete movement model. Using a conditional movement model specifically designed for generating random trajectories from template empirical trajectories (Technitis et al. 2016), we developed this approach with steppingstone migrations and similar movement strategies in mind. We extend this movement model to represent the two major states of stepping-stone migrations, the non-stop migratory flights and the staging periods, using a stochastic switch informed by empirical estimates of typical duration of both behaviours. Our multi-state movement model can simulate migratory trajectories that realisticically represent empiricallycollected migratory movements by exclusively sampling from empirical distribution functions. We develop a measure of route viability that integrates properties of the simulated trajectory and its environmental context to assess the joint suitability of the simulated migratory route and timing strategy. For stepping-stone migrations, we assume that the quality of stopover sites between the breeding grounds and wintering areas predominantly determines how preferable a certain route might be (Green et al. 2002; Drent et al. 2007). While the migration simulation model and the measure of route viability we introduce here are tailored for our study system, the approach in general is flexible and could be applicable to many other study systems and strategies. 
Specifically, we apply the conditional movement model on a pronounced long-distance migrant, the barheaded goose (Anser indicus, Latham 1790). This species of waterbird occurs in Central Asia and is well known for its incredible performance of crossing the Himalayas during migration. The distribution range of bar-headed geese is characterised by four distinct breeding areas which are mirrored by four distinct wintering areas south of the Himalayas. Previous tracking studies have revealed that large parts of the respective populations migrate from their breeding grounds in Mongolia, northern China and the Tibetan Plateau over the Himalayas to their wintering grounds on the Indian subcontinent (e.g., Bishop et al. 1997; Takekawa et al. 2009; Guo-Gang et al. 2011; Hawkes et al. 2011; Prosser et al. 2011). But while the crossing of the Himalayas has been studied in great detail (Hawkes et al. 2011, 2013; Bishop et al. 2015), less is known about the connectivity between range fragments both within the wintering and breeding range (Takekawa et al. 2009). The bar-headed goose thus provides a suitable study species for our approach. We establish a model for bar-headed goose migrations from previously published tracking data, and simulate migrations of unobserved individuals between all fragments of the species' distribution range. We assess the viability of these trajectories during several times of year using a segmented habitat suitability model to derive a dynamic migratory connectivity network. To assess whether this migratory connectivity network could serve as a quantitative null hypothesis for barheaded goose migration, we test our predictions against two very simple hypotheses generated from previously published studies.

Stable isotope analyses suggested that the connectivity within the breeding range of bar-headed geese is relatively high (Bridge et al. 2015), a notion that has been supported by tracking data as well (Cui et al. 2010). In the wintering range, however, relatively few movements have been observed (Kalra et al. 2011). Based on these findings (Cui et al. 2010; Kalra et al. 2011; Bridge et al. 2015), we expect to find a higher overall viability of trajectories between the fragments of the breeding range than within the wintering range. We further predict that on average, the temporal variation in viability of simulated migratory routes within the breeding grounds should be higher than within the wintering grounds. Overall, we would like to introduce a new approach for deriving environmentally informed quantitative null hypotheses for animal movement which can be utilised for estimating migratory connectivity based on limited observations (summarised in Fig. 1).

\section{Methods}

Tracking data and movement model

Tracking data of bar-headed geese were available to us from a broader disease and migration ecology study

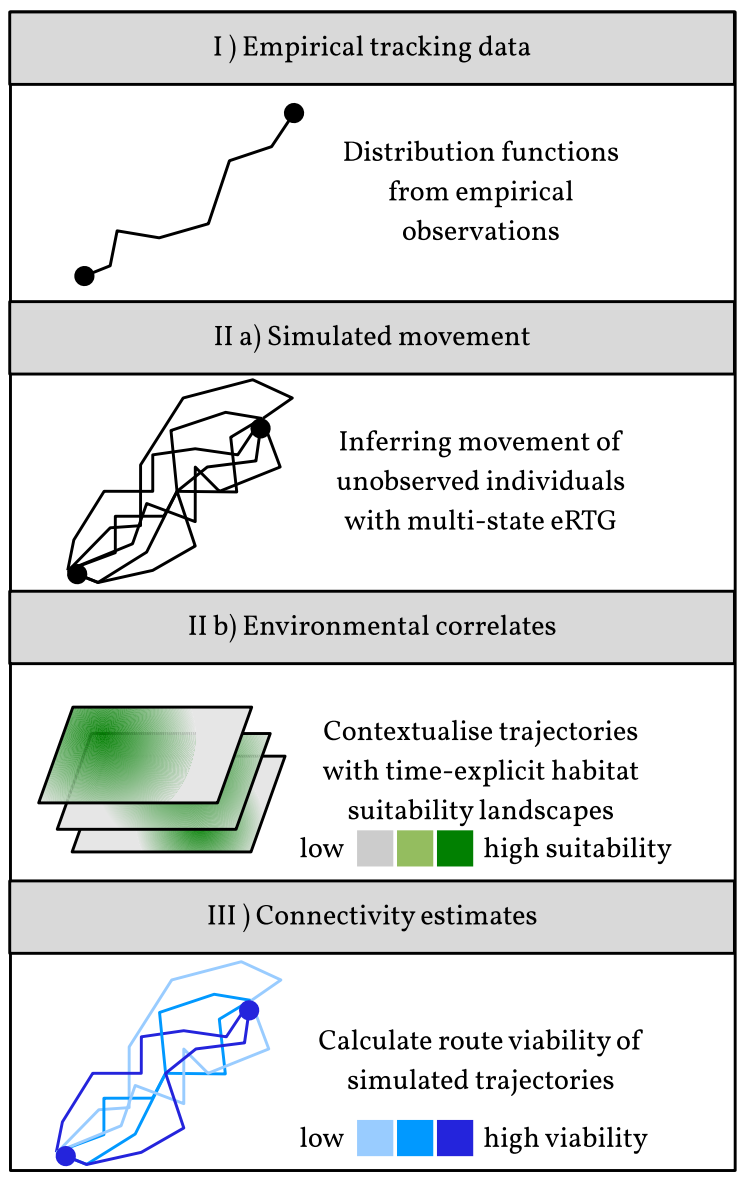

Fig. 1 General concept for our approach of environmentally informing simulated stepping-stone migrations: (I) Empirical tracking data are (IIa) used to derive an informed eRTG to simulate conditional movement between sites of interest, and (IIb) combined with environmental correlates to derive predictions of relevant measurements of landscape permeability (here: suitability of stopover sites). (III) Finally, the simulated conditional trajectories are evaluated based on characteristics of the trajectory and permeability using an informed measure of route viability 
implemented by the Food \& Agriculture Organization of the United Nations (FAO) and U.S. Geological Survey (USGS). In total, 91 individuals were captured during the years 2007-2009 in several locations: Lake Qinghai in China (hereafter termed "Lake Qinghai"), Chilika Lake and Koonthankulum bird sanctuary in India (hereafter termed "India"), and Terkhiin Tsagaan Lake, Mongolia (hereafter termed "West Mongolia"). All individuals were equipped with ARGOS-GPS tags which were programmed to record the animals' location every $2 \mathrm{~h}$ (ARGOS PTT-100; Microwave Telemetry, Columbia, Maryland, USA). Eighty of the deployed tags collected and transmitted data for $241 \pm 253$ (mean $\pm \mathrm{SD}$ ) days. In total, 169, 887 fixes could be acquired over the course of the tracking period (Table 1 and Takekawa et al. 2009; Hawkes et al. 2011). Individuals that were tracked for less than a complete year were excluded from the subsequent analyses, which left a total of 66 individuals (Lake Qinghai: 20, India: 20, West Mongolia: 26). We pooled data from all capture sites for the analyses.

We used the recently developed the empirical Random Trajectory Generator (eRTG, Technitis et al. 2016) to simulate the migrations of unobserved individuals of bar-headed geese. This movement model is conditional, i.e. simulates the movement between two end locations with a fixed number of steps based on a dynamic drift derived from a stepwise joint probability surface. One main advantage of the eRTG is that the trajectories it simulates retain the geometric characteristics of the empirical tracking data (step length, turning angle, as well as covariance and auto-correlation of step length and turning angle), as it relies entirely on empirical distribution functions. Consequently, if a destination cannot be reached within the realms of the empirical distributions of e.g. step lengths and turning angles, the simulation fails rather than forcing the last step towards the destination.

We extended this movement model by incorporating a stochastic switch between the two main states of bar-headed goose migration, non-stop migratory flights ("migratory state") and movements during staging periods at stopover locations ("stopover state"). We classified the entire tracking data according to the individuals' movement behaviour to identify these states prior to extracting the empirical distributions functions for the eRTG. First, we clustered the locations in the tracking data using an expectationmaximisation binary clustering algorithm designed for annotating animal movement data (EMbC, Garriga et al. 2016). The EMbC divided the trajectories of barheaded geese into four behavioural classes (slow speed \& low turning angles, slow speed \& high tuning angles, high speed \& low turning angles, and high speed \& high turning angles), which we then reclassified into two behavioural classes, namely highspeed movements (combining the two high speed classes) and low-speed movements (combining the two low speed classes). Within the high-speed behavioural cluster, the average speed between locations was $8.4 \pm 6.7 \frac{\mathrm{m}}{\mathrm{s}}$ (mean $\pm \mathrm{SD}$ ) whereas the average speed for the low-speed behavioural cluster was $0.3 \pm 1.0 \frac{m}{s}$ (mean $\pm \mathrm{SD}$ ). As estimates of speed and turning angle are highly dependent on the sampling rate of the data, we removed those parts of the trajectories that exceeded the average sampling interval of $2 \mathrm{~h}$. Subsequently, we used the low-speed locations for the empirical distribution functions for the stopover state of the two-state eRTG, and the

Table 1 A summary of the catching sites and corresponding sample sizes

\begin{tabular}{llllll}
\hline Capture site & Year of capture & Sample size(individuals) & First fix & Tracking days & GPS fixes \\
\hline Lake Qinghai & 2007 & 13 & Mar 25-31 & $303[207 ; 411]$ & $1,670[682 ; 2,565]$ \\
& 2008 & 10 & Mar 30-Apr 4 & $396[260 ; 845]$ & $2,211[1,341 ; 3,573]$ \\
India & 2008 & 17 & Dec 10-18 & $129[92 ; 401]$ & $2,060[1,578 ; 2,714]$ \\
& 2009 & 7 & Jan 27 - Feb 06 & $134[53 ; 448]$ & $1,321[1,107 ; 3,800]$ \\
West Mongolia & 2008 & 19 & Jul 13-15 & $122[90 ; 190]$ & $537[366 ; 1,312]$ \\
& 2009 & 14 & Jul 05-08 & $105[100 ; 128]$ & $421[330 ; 473]$ \\
\hline
\end{tabular}

The number of tracking days and GPS fixes are listed as a median per individual, with the 25 and $75 \%$ quantiles in square brackets. Eleven out of the total of 91 tags deployed did not transfer any data, and are not included in this table 
locations classified as high-speed for the empirical distribution functions for the migratory state of the eRTG (see Figure S2). Finally, we derived the step lengths and turning angles from each coherent stretch of data (i.e. only subsequent fixes with a sampling rate of 2 hours). Following this, we calculated the changes in step length and turning angle at a lag of one observation, as well as the covariance between contemporary observations of step length and turning angle. We derived the corresponding empirical distribution functions for both movement states and prepared them for use in the eRTG functions.

Finally, we determined the duration of staging periods, and the duration and cumulative distance of individual migratory legs from the tracking data. We first identified seasonal migration events between breeding and wintering grounds (and vice versa) in the empirical trajectories using the behavioural annotation. We then determined migratory legs (sequential locations classified as migratory state) as well as stopovers (sequential locations classified as stopover state, with a duration $>12 h$ ). We used two main proxies to characterise migratory legs, namely cumulative migratory distance as well as duration, and one proxy to characterise staging periods, namely stopover duration. We calculated these proxies for all individuals and migrations, and determined the maximum observed distance $\left(\mathrm{dm}_{\max }\right)$ and duration $\left(\mathrm{Tm}_{\max }\right)$ of a migratory leg. As we did not distinguish between extended staging (e.g. during moult, or after unsuccessful breeding attempts) from use of stopover locations during migration, we calculated the $95 \%$ quantile of the observed stopover durations $\left(\mathrm{Ts}_{\max }\right)$ rather than the maximum.

\section{Simulating a bar-headed goose migration with the two-state eRTG}

When simulating a conditional random trajectory between two arbitrary locations $a$ and $z$, the two-state eRTG initially draws from the distribution functions for the migratory state, producing a fast, directed trajectory. To determine the time available for moving from $a$ to $z$, we assumed the mean empirical flight speed derived for the migratory state, and calculated the number of required steps accordingly. While simulating the trajectory, after each step modelled by the eRTG, the cumulative distance of the trajectory as well as the duration since the start of the migratory leg were calculated. By using cumulative distance and duration as well as the empirically derived $\mathrm{dm}_{\max }$ and $\mathrm{Tm}_{\max }$, our two-state eRTG was based on a binomial experiment with two possible outcomes: switching to the stopover state with a probability of $p_{m s}$, or resuming migration with a probability of $1-p_{m s}$. We defined $p_{m s}$, the transition probability to switch from migratory state to stopover state, as

$p_{m s}(t)=\frac{\sum_{i=0}^{t}(d m)}{d m_{\max }} \times \frac{\sum_{i=0}^{t}(T m)}{T m_{\max }}$

where $\mathrm{dm}$ and $\mathrm{Tm}$ represent the distance and duration between two consecutive locations during a migratory leg. At step $t$, the simulation of the migratory movement can switch to the unconditional stopover state, corresponding to a correlated random walk, with a probability of $p_{m s}(t)$. Likewise, the simulation can switch back from stopover state to migratory state with the probability $p_{s m}(t)$, which we defined as as

$p_{s m}(t)=\left(\frac{\sum_{i=0}^{t}(T s)}{T s_{\max }}\right)^{2}$

where Ts represents the duration between two consecutive locations during a stopover. This process is then repeated until the simulation terminates because: either the trajectory reached its destination, or the stepwise joint probability surface did not allow for reaching the destination with the remaining number of steps (resulting in a dead end or zero probability).

Evaluating the plausibility of simulated migrations

We estimated the plausibility of each simulated trajectory, representing a unique migratory route, using a measure we called route viability $\Phi$ aimed to integrate the ecological context into the movement simulations. We developed this measure specifically with the stepping-stone migratory strategy of barheaded geese or similar species in mind, and it is defined by the time spent in migratory mode, the time spent at stopover sites, and the habitat suitability of the respective utilised stopover sites. For this specific measure of route viability, we made two main assumptions: (1), it is desirable to reach the destinations quickly, i.e. staging at a stopover site comes at the cost of delaying migration, and (2), the cost imposed by delaying migration is inversely-proportional to the quality of the stopover site, i.e. the use of 
superior stopover sites can counterbalance the delay. Our argument for these assumptions is that during spring migration, the arrival at the breeding grounds needs to be well-timed with the phenology of their major food resources (Bauer et al. 2008). Furthermore, the quality of stopover sites has been shown to be of crucial importance for other species of geese with similar migratory strategies (Green et al. 2002; Drent et al. 2007).

Each simulated multi-state trajectory between two arbitrary locations $a$ and $z$ can be characterised by a total migration duration $\tau_{a, z}$, which consists of the total flight time $\tau_{M, a, z}$ and the total staging time at stopover sites $\tau_{S, a, z}$. The total flight time $\tau_{M, a, z}$ is the sum of the time spent flying during each migratory leg $l$, and is thus $\tau_{M, a, z}=\sum_{l=0}^{n} t_{M}(l)$, with $t_{M}(l)$ corresponding to the time spent flying during migratory leg $l$. Similarly, the total staging time $\tau_{S, a, z}$ consists of the staging times at all visited stopover sites, corresponding to $\tau_{S, a, z}=\sum_{k=0}^{n} t_{S}(k)$, where $t_{S}(k)$ amounts to the staging time at stopover site $k$. For our metric of route viability, we will consider the time spent staging at stopover locations $\tau_{S, a, z}$ as a delay compared to the time spent in flight. This delay is, however, mediated by the benefit $b$ an individual gains at the stopover site from replenishing its fat reserves. We define this benefit gained by staying at stopover site $k, b(k)$, as proportional to the time spent at site $k, t_{S}(k)$, and the habitat suitability of site $k, S(k)$. This habitat suitability $S$ should range between $[0,1]$, which allows our measure of route viability to range between $[0,1]$ as well. We further assume the effects of several sequential stopovers to be cumulative, and thus define the total benefit of a migratory trajectory between locations $a$ and $z$ with $n$ stopovers as $B_{a, z}=\sum_{k=0}^{n} S(k) \times t_{S}(k)$. Finally, we define the route viability $\Phi_{a, z}$ of any trajectory between $a$ and $z$ as:

$$
\Phi_{a, z}=\frac{\tau_{M, a, z}}{\tau_{M, a, z}+\tau_{S, a, z}-B_{a, z}}=\frac{\tau_{M, a, z}}{\tau_{a, z}-B_{a, z}}
$$

Thus, the viability of a trajectory with no stopovers and a trajectory with stopovers of the highest possible quality $(S(k)=1)$ will be equal, and is defined solely by the time the individual spent in migratory state $\left(\Phi_{a, z}=1\right)$. For trajectories with stopovers in less than optimal sites, however, the viability of trajectories is relative to both the staging duration and quality of stopover sites, and should take values of
$\frac{\tau_{M, a, z}}{\tau_{a, z}}<\Phi_{a, z}<1$. Using this metric, we assessed simulated trajectories in a way that is biologically meaningful for bar-headed geese. In the next section, we detail how we calculated the route viability $\Phi$ for each simulated migration.

A migratory connectivity network for bar-headed geese

We simulated migrations of bar-headed geese within the native range of the species which naturally occurs in Central Asia $\left(68-107^{\circ} \mathrm{N}, 9-52^{\circ} \mathrm{E}\right)$. According to BirdLife International and NatureServe (2013), both the breeding and wintering range are separated into four distinct range fragments (see also Figure S1), with minimum distances between range fragments ranging from $79 \mathrm{~km}$ to $2884 \mathrm{~km}$. For this study, we investigated how well, in terms of an environmentally informed measure of route viability and the number of stopovers required to reach a range fragment, these range fragments can be connected by simulated migrations of bar-headed geese.

To choose start- and endpoints for the simulated migrations, we sampled ten random locations from each of the range fragments indicated in the distribution data provided by BirdLife International and NatureServe (2013). We simulated 1000 trajectories for all pairs of range fragments (100 trajectories per location pair) and counted the number of successes (trajectories reach the destination) and failures (trajectories terminate in a dead end). We proceeded to calculate the viability of simulated routes in the following way: Initially, we determined the total duration of the trajectory between locations $a$ and $z$, $\tau_{a, z}$, the number of stopover sites used, $n_{a, z}$, as well as the time spent at each stopover site, $t_{S}(k)$, for each of the total $n_{a, z}$ stopovers (corresponding to the number of steps multiplied with the location interval of $2 \mathrm{~h}$ ). We determined the habitat suitability of stopover locations $S(k)$ using habitat suitability landscapes for bar-headed geese during five periods of the year (see Figure S3): winter/early spring (mid-November-February), mid-spring (mid March-mid April), late spring/summer (mid April-mid August), early autumn (mid August-mid September), and late autumn (mid September-mid November). We identified these periods using a segmentation by habitat use (van Toor et al. 2016, for details see Section A in the Electronic 
Supplementary Material (ESM)). The segmentationby-habitat-use procedure uses animal location data and associated environmental information to identify time periods for which habitat use is consistent. Habitat suitability models derived for these time periods should thus reflect differences in habitat use by bar-headed geese throughout the year. We used time series of remotely sensed environmental information and Random Forest models (Breiman 2001) to derive habitat suitability models corresponding to these five time periods, and predicted the corresponding habitat suitability landscapes (Section $\mathrm{A}$ in the ESM). Following the prediction of habitat suitability landscapes for winter/early spring, mid-spring, late spring/summer, early autumn, and late autumn, we annotated all stopover state locations of the simulated trajectories with the corresponding habitat suitability. We then calculated the benefit $b$ gained by using a stopover location $k$ using the mean suitability for each of the stopover locations, $S(k)$, and the duration spent at stopover locations, $\tau_{S}(k)$.

To calculate the route viability $\Phi_{a, z}$, we also required an estimate for duration of migration if a simulation were exclusively using the migratory state $\tau_{M, a, z}$, without the utilisation of stopover sites. We used a simple linear model to predict flight time as a function of geographic distance which we trained on the empirical data derived from the migratory legs (see Section B in the ESM for details). By basing the linear model on the empirical migratory legs rather than mean flight speed, the estimate for $\tau_{M}$ retains the inherent tortuosity of waterbird migrations. For each simulated trajectory, we then calculated the geographic distance between its start- and endpoint, and predicted the expected flight time $\tau_{M, a, z}$. Finally, we calculated route viability $\Phi_{a, z}$ for all trajectories using Eq. 3, repeating the process for each of the five suitability landscapes derived from the segmentation by habitat use. This resulted in five different values of $\Phi_{a, z}$ for every simulated trajectory, corresponding to winter/early spring, mid-spring, late spring/summer, early autumn, and late autumn, respectively.

\section{Calculating migratory connectivity as average route viability}

We calculated migratory connectivity between range fragments as the average route viability $\Phi_{\text {avg. }}$ of all trajectories connecting two range fragments. We calculated this average by using non-parametric bootstrapping on the median route viability $\Phi_{\text {avg }}$. (using 1000 replicates), and also computed the corresponding $95 \%$ confidence intervals (CI) of the median route viability $\Phi_{\text {avg. }}$. We did this for each of the five time periods represented in the suitability landscapes, and also computed an overall migratory connectivity by averaging all five habitat suitability values for each stopover site prior to calculating $\Phi$.

We wanted to compare migratory connectivity within the breeding range and migratory connectivity in the wintering range to test our first hypothesis stating that migratory connectivity should be higher within the breeding range. To do so, we differentiated between route viability among breeding range fragments $\left(\Phi_{\text {breeding }}\right)$, among the wintering areas ( $\left.\Phi_{\text {wintering }}\right)$, and between breeding and wintering range fragments $\left(\Phi_{\text {mixed }}\right)$. We computed the median and $95 \%$ CIs of route viability with non-parametric bootstrapping with 1000 replicates, using the average habitat suitability of all five suitability landscapes for all trajectories within the breeding range, all trajectories in the wintering range, and all trajectories connecting breeding range fragments with wintering range fragments.

To test our second hypothesis, stating that variation in migratory connectivity throughout the year should be higher in the breeding range than in the wintering range, we calculated the standard deviation of route viability for the five suitability landscapes in the breeding range and in the wintering range. We did this by again differentiating trajectories in the wintering range, trajectories in the breeding range, and trajectories connecting breeding range fragments with wintering range fragments. We computed route viability $\Phi$ for each of the five suitability landscapes for all trajectories, and pooled the corresponding values for $\Phi_{\text {late winter/early spring }}, \Phi_{\text {mid-spring }}, \Phi_{\text {late spring/summer }}$, $\Phi_{\text {early autumn }}$, and $\Phi_{\text {late autumn }}$ for the wintering range, for the breeding range, and for trajectories connecting breeding range fragments with wintering range fragments separately. We then used a non-parametric bootstrapping (1,000 replicates) on the standard deviation over the five time periods, and determined the corresponding $95 \%$ CIs on the standard deviation. 


\section{Calculating route viability for empirical migrations}

Following the above described procedure, we annotated the stopover locations of empirical migrations with the habitat suitability of the corresponding time period, and calculated the route viability for these migratory trajectories in the same way as described above. We then used non-parametric bootstrapping on the median route viability for all empirical migrations $\left(\Phi_{\text {emp.total }}\right)$, only spring migrations $\left(\Phi_{\text {emp.spring }}\right)$ and only autumn migrations ( $\left.\Phi_{\text {emp.,autumn }}\right)$, and computed 95\% CIs for the median of $\Phi_{\text {emp.total }}, \Phi_{\text {emp.,spring }}$, and $\Phi_{\text {emp.,autumn }}$.

\section{Results}

Route viability of empirical and simulated migrations

The simulations resulted in a total of 30,730 simulated trajectories, of which 8945 trajectories connected breeding range fragments (simulation success rate: $74.5 \%), 5393$ trajectories connected wintering range fragments (simulation success rate: $44.9 \%$ ), and the remaining 16,392 trajectories connected breeding and wintering range fragments (simulation success rate: $51.2 \%$; see Figure S4). While all these trajectories were successful in connecting origin and destination (i.e. did not result in a dead end), they differed profoundly in their route viability $\Phi_{\text {simulated, }}$ which ranged between 0.014 and 0.59 . We found that simulated migrations had a higher route viability for late spring and summer than for autumn (Fig. 2).

The range of route viability for simulated migrations was comparable to that of the empirical migrations ( $\Phi_{\text {emp.total }}: 0.01-0.38$ ). Overall, we found that route viability of empirical migrations was higher for spring migrations $\left(\Phi_{\text {emp.,spring }}\right.$ : $[0.0614 ; 0.1070] ; 95 \%$ CIs on the median) than for autumn migrations ( $\Phi_{\text {emp.,autumn: }}$ [0.0270; 0.0514]; 95\% CIs on the median). This was caused both by differences in the habitat suitability of utilised stopover locations and by differences in migration duration between spring and autumn migrations. We found that bar-headed geese on average stayed longer at stopover locations during autumn than during spring migrations (spring: $6.8 \pm$ 14.2 days, autumn: $11.8 \pm 12.2$ days; mean \pm SD).
Migratory connectivity network informed by route viability

We separated the simulated trajectories into movements within the breeding range, movements within the wintering range, and movements resembling seasonal migrations between the breeding and wintering range. Here, we found that viability of trajectories was highest within the breeding range $(95 \%$ CIs for median $\Phi_{\text {breeding }}:[0.0676 ; 0.0684] ; 95 \%$-quantiles of median $\left.\Phi_{\text {breeding }}:[0.1469 ; 0.1546]\right)$, and lowest within the wintering range $(95 \%$ CIs for median $\Phi_{\text {wintering }}:[0.0590 ; 0.0596]$; 95\%-quantile of median $\left.\Phi_{\text {wintering }}:[0.1090 ; 0.1147]\right)$, predicting that movements between range fragments should occur more often within the breeding than in the wintering areas. The median route viability for migrations between breeding and wintering range fragments was intermediate $\left(95 \%\right.$ CIs for median $\Phi_{\text {mixed }}:[0.0618 ; 0.0622]$; $95 \%$-quantile of median $\Phi_{\text {mixed }}:[0.1224 ; 0.1296]$ ). These patterns are reflected in the simplified network of average migratory connectivity $\Phi_{\text {avg. }}$ (Fig. 3). We also identified the single trajectory with the maximum route viability between range fragments rather than the median (Figure S5). This network of maximum migratory connectivity shows that migrations that connect the breeding and wintering ranges have the highest route viability. Finally, the number of stopover locations of movements was proportional to the geographic distance between range fragments (Figure S6).

Temporal variability of migratory connectivity

We found that the spatial patterns of migratory connectivity varied across the five habitat suitability landscapes representing five periods of consistent habitat suitability (Fig. 4; see also Figure S3 for details on the temporal correspondence of the time periods). For the suitability landscapes derived for winter/early spring, mid spring, and late spring/summer, the estimated connectivity predicted that bar-headed goose migrations are most likely to occur between the wintering and breeding range, and within the breeding range. For early autumn, connectivity patterns predicted that movement is most likely between breeding and wintering areas. For late autumn, we observed connectivity within the wintering range of 


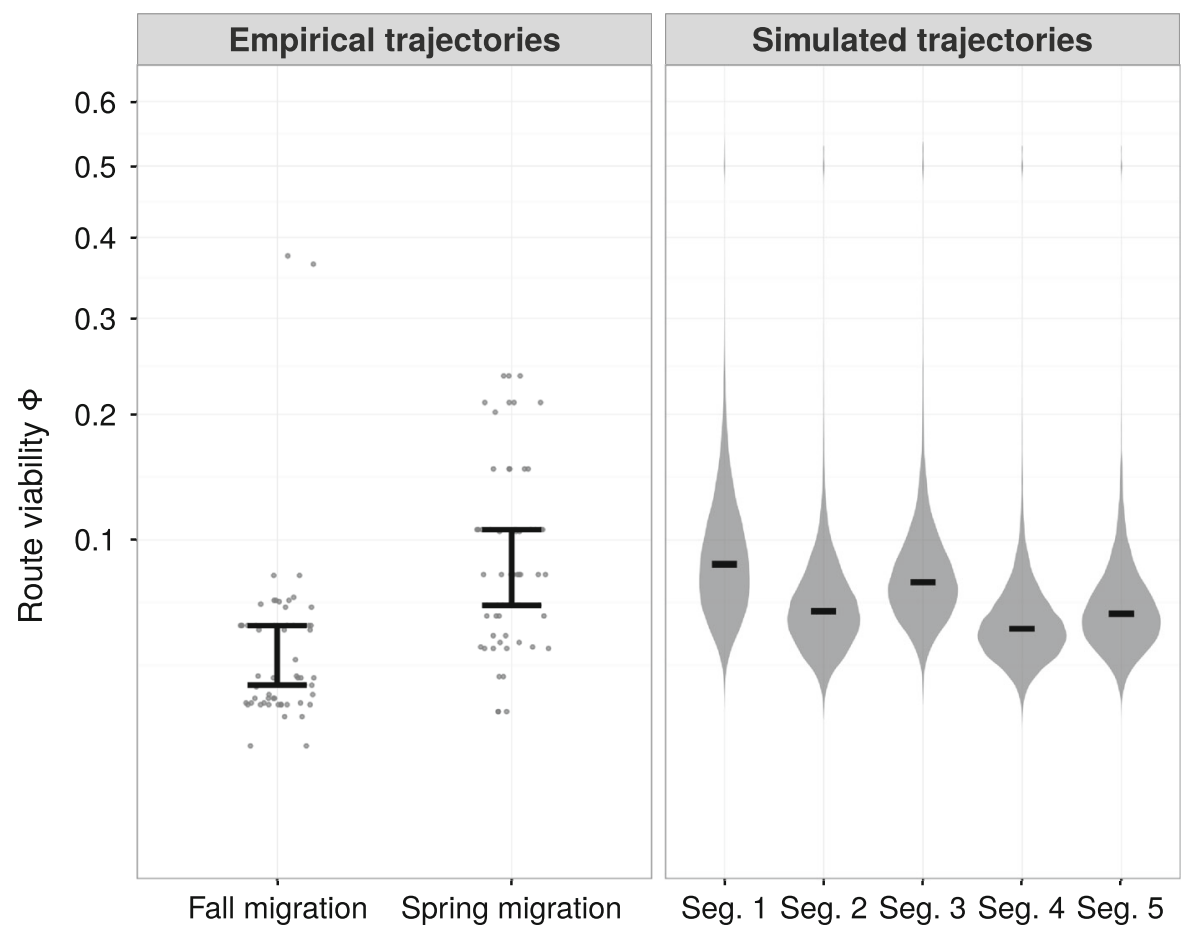

Fig. 2 The route viability $\Phi$ of empirical and simulated migrations. Here we show $\Phi$ for spring and autumn migrations, as well as the $\Phi$ for the simulated trajectories across all five suitability landscapes (Seg. 1: winter/early spring, Seg. 2: midspring, Seg. 3: late spring/summer, Seg. 4: early autumn, Seg. 5:

the species. We calculated the $95 \%$ CIs for the overall migratory connectivity values for each time period (Fig. 2), which predicted the highest median route viability for the periods from winter/early spring (midNovember-February) as well as from late spring/summer (mid April-mid August). We also compared the standard deviation of route viability across suitability landscapes and found the highest variation for the breeding range $\left(95 \%\right.$ CIs for s.d. of $\Phi_{\text {breeding: }}$ : $[0.0124 ; 0.0133])$ and the lowest variation for the wintering range $(95 \% \mathrm{CIs}$ for s.d. of $\left.\Phi_{\text {wintering }}:[0.0041 ; 0.0046]\right)$. Again, the trajectories between breeding and wintering range fragments showed intermediate values $(95 \%$ CIs for s.d. of $\Phi_{\text {mixed }}$ : [0.0084; 0.0089]).

\section{Discussion and conclusions}

Using tracking data of bar-headed geese and the empirical Random Trajectory Generator (eRTG), we late autumn). The black bars show the $95 \%$ CIs for the respective medians, and the grey dots and violin plots show the observed (empirical trajectories) and route viability densities (simulated trajectories)

were able to successfully develop a model that simulates the migratory movements of bar-headed geese. Our extension of the eRTG with a stochastic switch between a migratory state and a stopover state was sufficient to capture the overall migratory strategy of this species. With this model for bar-headed goose migrations, we inferred the migrations of unobserved individuals between all fragments of the species' distribution range, and used an environmentallyinformed measure of route viability to derive average estimates of migratory connectivity between range fragments. We put this simplified predictive network of migratory connectivity to a simple test using predictions derived from the literature. Indeed, we found that the average route viability, as an indicator of migratory connectivity, was higher within the species' breeding range ( $\left.\Phi_{\text {breeding }}\right)$ than in the wintering areas $\left(\Phi_{\text {wintering }}\right)$, confirming the expectations from the literature (Cui et al. 2010; Kalra et al. 2011; Bridge et al. 2015). While bar-headed geese are thought to be philopatric to their breeding grounds 


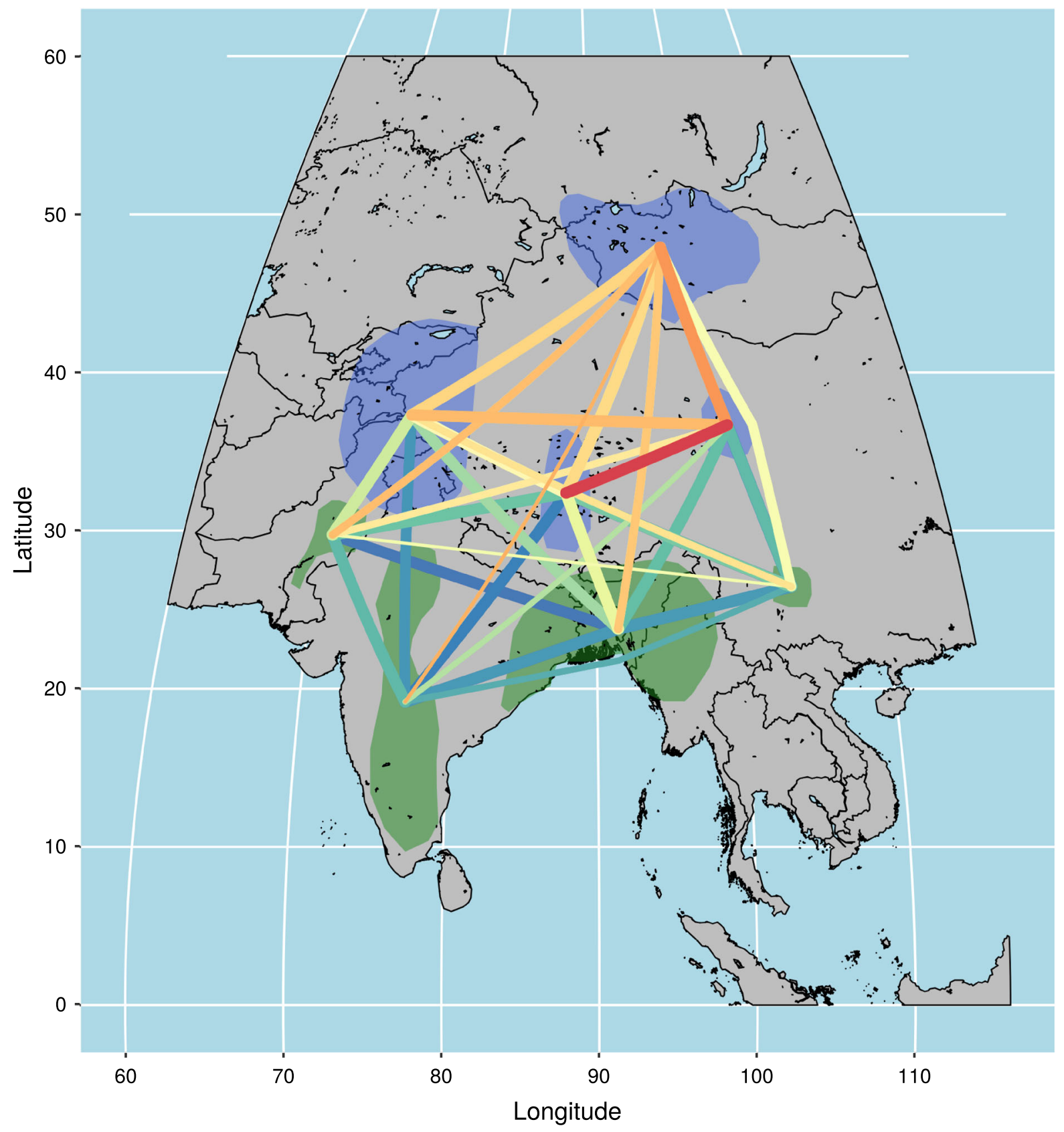

Median route viability $\Phi$

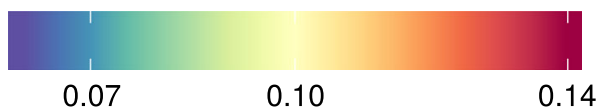

Number of trajectories

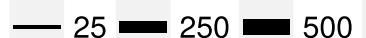

750
Fig. 3 The median route viability $\Phi$ between range fragments of bar-headed geese. We summarised $\Phi$ for all pairwise range fragment trajectories using the median route viability. The thickness of edges represents the sample size. Blue polygons show the native breeding area of the species. Green polygons show the native wintering range. Long edges are curved for sake of visibility 


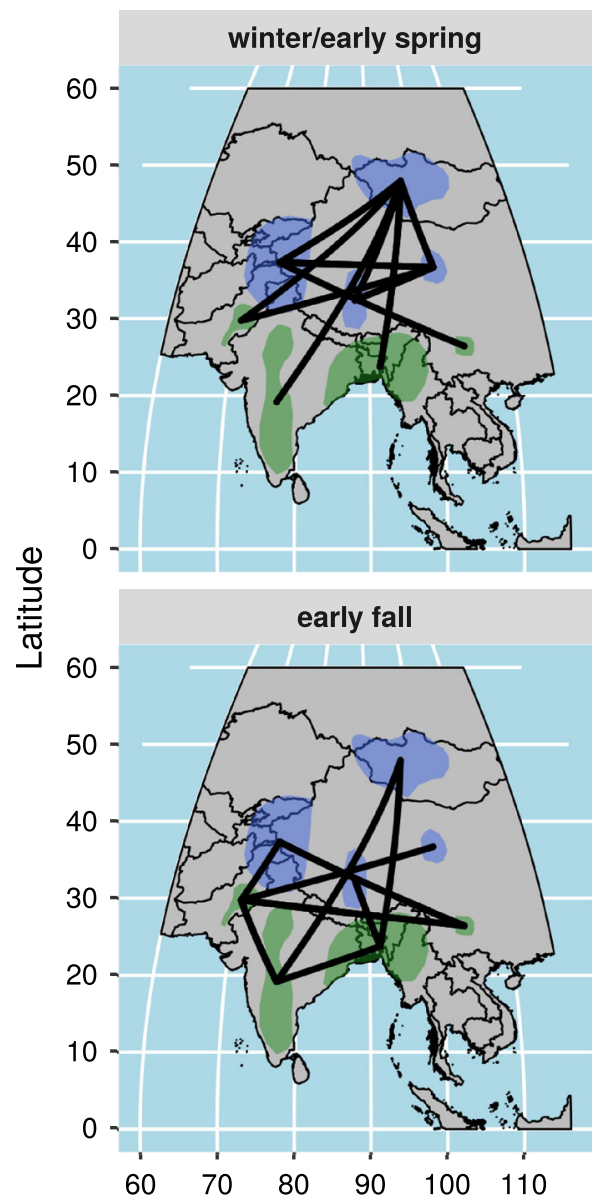

Fig. 4 Temporal dynamics of the route viability $\Phi$. Here we show the predicted movements for each of the five suitability landscapes separately. The visible edges of the network have a

(Takekawa et al. 2009), the post-breeding period seems to be a time of great individual variability and extensive movements (Cui et al. 2010). This flexibility in long-distance movements has also been observed for other Anatidae species (e.g., Gehrold et al. 2014), and due to the temporary flightlessness during moult the choice of suitable moulting sites is critical to many waterfowl species. As the average route viability within the breeding range and during the summer months is high, unsuccessful breeders and individuals in the post-breeding period may not be not limited by sufficiently suitable stopover locations when moving between breeding range fragments. Furthermore, our results confirmed that the temporal variability of migratory connectivity was higher in the

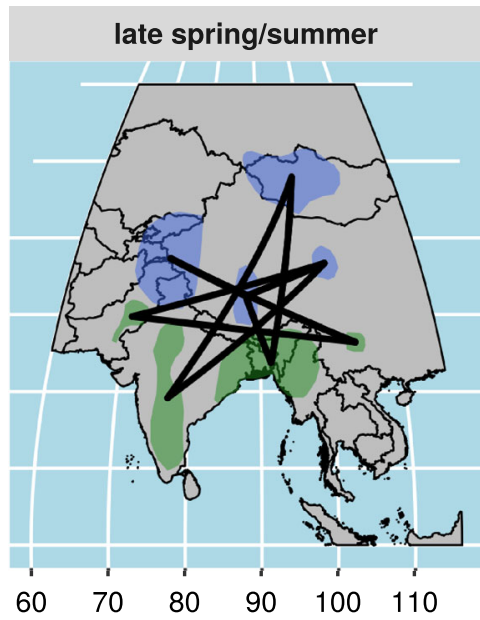

late fall

60 
even without additional filtering. Consequently, in combination with relevant environmental and ecological information, the simulation of unobserved migrations using a model like our two-state eRTG may provide a sensible and quantitative null hypothesis for the migrations of bar-headed geese or species with similar strategies. While we determined the route viability using only the habitat suitability of the stopover locations and measures of migratory duration, other correlates such as wind support or altitude profile may easily be incorporated for the migratory state. Similarly, the transition probabilities that mediate the switch between movement modes may be extended to include environmental conditions. In general, our stochastic switch performed reasonably well in replicating the movement behaviour observed from recorded tracks. We used simple functions to determine transition probabilities due to the long fix interval $(2 \mathrm{~h})$ and the amount of missed fixes in the data. If a larger sample size were available, the functions we used (see Eqs. 1, 2) could be replaced by a probability distribution function that more adequately represents the decision-making of bar-headed geese. Alternatively, algorithms such as state-space models could be integrated to simulate animal movement with a more complex configuration of movement states (Morales et al. 2004; Patterson et al. 2008). With a few modifications specific to the species of interest, the approach described in this study could be adapted for other scenarios of animal movement. One important application for our approach could be to support capture-mark-recapture data, especially when tracking data for multiple individuals are hard to acquire. Simulations from a multi-state movement model informed by the movements of a few representative individuals could be used to infer alternative routes connecting the re-sightings of individually marked animals. A corresponding relevant measure of route viability could then be used to explore alternative strategies from an ecologically informed perspective. In such a study, it could also be of interest to use Bayesian approaches to approximate ideal routes using the environmental context.

Furthermore, our results highlight the importance of integrating temporal changes in habitat use of moving animals into measures of landscape connectivity. Zeigler and Fagan (2014) argue that the ecological function of landscape connectivity through animal movement is not only determined by where, but also when the environment provides the conditions that allow an individual to move from $a$ to $z$. In our study, estimates of migratory connectivity were affected by changes in the predicted habitat suitability of stopover locations, whereas in other cases, changes in vegetation density throughout the year or the temporary freezing of waterbodies can be imagined to change connectivity between distant sites. Using time series of environmental information in combination with an approach that segments a species utilisation of the environment for moving, as shown here, could help with the identification of temporal patterns of landscape connectivity. Accounting for such temporal changes in connectivity could also help to better understand how for example, diseases can spread through through a metapopulation (such as white-nose syndrome, Blehert et al. 2009; Turner et al. 2011, or Influenza A viruses in birds, Gaidet et al. 2010; Newman et al. 2012).

Overall, models that incorporate a species' movement behaviour and its utilisation of the environment can provide sensible estimates for landscape connectivity. This approach possibly provides the basis for a wider range of applications, for example the estimation of seed or pathogen dispersal on a population level. Our approach provides a starting point for complementing tracking efforts with ecologically relevant estimates of a species' potential to migrate through a landscape and act as a link between patches, populations, and ecosystems.

Acknowledgements Open access funding provided by Max Planck Society. This work was made possible by the efforts of the many cooperating scientists in China, India, and Mongolia who assisted the Wildlife Health and Ecology Program at the Food and Agriculture Organization of the United Nations (FAO) and US Geological Survey (USGS) in the collection of the tracking data with ecological findings provided in other reports and papers. We are indebted to Markus Rampp and Karin Gross at the Computation Center of the Max Planck Society for their support with the computational facilities. We would like to thank Todd Lookingbill and one anonymous reviewer for their valuable comments. The use of trade, product, or firm names in this publication is for descriptive purposes only and does not imply endorsement by the U.S. Government or the FAO. The views expressed in this publication are those of the author(s) and do not necessarily reflect the views or policies of the FAO. This study re-analysed several datasets from FAO and USGS published studies; Institutional Animal Care and Use Committee details are available in the original publications. MLvT was supported by the International Max Planck Research School for Organismal Biology and the European Union's 
Horizon 2020 research and innovation programme under Grant Agreement No. 727922 (Delta-Flu).

Open Access This article is distributed under the terms of the Creative Commons Attribution 4.0 International License (http:// creativecommons.org/licenses/by/4.0/), which permits unrestricted use, distribution, and reproduction in any medium, provided you give appropriate credit to the original author(s) and the source, provide a link to the Creative Commons license, and indicate if changes were made.

\section{References}

Almpanidou V, Mazaris AD, Mertzanis Y, Avraam I, Antoniou I, Pantis JD, Sgardelis SP (2014) Providing insights on habitat connectivity for male brown bears: a combination of habitat suitability and landscape graph-based models. Ecol Model 286:37-44

Altizer S, Bartel R, Han BA (2011) Animal migration and infectious disease risk. Science 331:296-302

Austin D, Bowen WD, McMillan JI (2004) Intraspecific variation in movement patterns: modeling individual behaviour in a large marine predator. Oikos 105:15-30

Bauer S, Gienapp P, Madsen J (2008) The relevance of environmental conditions for departure decision changes en route in migrating geese. Ecology 89:1953-1960

Bauer S, Hoye BJ (2014) Migratory Animals Couple Biodiversity and Ecosystem Functioning Worldwide. Science 344:1242552

BirdLife International and NatureServe (2013) Bird species distribution maps of the world. Version 3

Bishop CM, Spivey RJ, Hawkes LA, Batbayar N, Chua B, Frappell PB, Milsom WK, Natsagdorj T, Newman SH, Scott GR, Takekawa JY, Wikelski M, Butler PJ (2015) The roller coaster flight strategy of bar-headed geese conserves energy during Himalayan migrations. Science 347:250-254

Bishop MA, Yanling S, Zhouma C, Binyuan G (1997) Barheaded Geese Anser indicus wintering in south-central Tibet. Wildfowl 48:118-126

Blehert DS, Hicks AC, Behr M, Meteyer CU, Berlowski-Zier BM, Buckles EL, Coleman JT, Darling SR, Gargas A, Niver R, Okoniewski JC, Rudd RJ, Stone WB (2009) Bat white-nose syndrome: an emerging fungal pathogen? Science 323:227-227

Breiman L (2001) Random forests. Mach Learn 45:5-32

Bridge ES, Kelly JF, Xiao X, Batbayar N, Natsagdorj T, Hill NJ, Takekawa JY, Hawkes LA, Bishop CM, Butler PJ, Newman SH (2015) Stable isotopes suggest low site fidelity in Bar-headed Geese (Anser indicus) in Mongolia: implications for disease transmission. Waterbirds 38:123-132

Calabrese JM, Fagan WF (2004) A comparison-shopper's guide to connectivity metrics. Front Ecol Environ 2:529-536

Codling EA, Plank MJ, Benhamou S (2008) Random walk models in biology. J R Soc Int 5:813-834

Cui P, Hou Y, Tang M, Zhang H, Zhou Y, Yin Z, Li T, Guo S, Xing Z, He Y, Prosser DJ, Newman SH, Takekawa JY, Yan B, Lei F (2010) Movement patterns of Bar-headed Geese
Anser indicus during breeding and post-breeding periods at Qinghai Lake, China. J Ornithol 152:83-92

Drent RH, Eichhorn G, Flagstad A, Graaf AJ, Litvin KE, Stahl J (2007) Migratory connectivity in Arctic geese: spring stopovers are the weak links in meeting targets for breeding. J Ornithol 148:501-514

Ferreras P (2001) Landscape structure and asymmetrical interpatch connectivity in a metapopulation of the endangered Iberian lynx. Biol Conserv 100:125-136

Fortin D, Beyer HL, Boyce MS, Smith DW, Duchesne T, Mao JS (2005) Wolves influence elk movements: behaviour shapes a trophic cascade in Yellowstone National Park. Ecology 86:1320-1330

Gaidet N, Cappelle J, Takekawa JY, Prosser DJ, Iverson SA, Douglas DC, Perry WM, Mundkur T, Newman SH (2010) Potential spread of highly pathogenic avian influenza H5N1 by wildfowl: dispersal ranges and rates determined from large-scale satellite telemetry. J Appl Ecol 47:1147-1157

Garriga J, Palmer JRB, Oltra A, Bartumeus F (2016) Expectation-maximization binary clustering for behavioural annotation. PLOS ONE 11:e0151984

Gehrold A, Bauer HG, Fiedler W, Wikelski M (2014) Great flexibility in autumn movement patterns of European gadwalls Anas strepera. J Avian Biol 45:131-139

Graham CH (2001) Factors influencing movement patterns of keel-billed toucans in a fragmented tropical landscape in Southern Mexico. Conserv Biol 15:1789-1798

Green M, Alerstam T, Clausen P, Drent R, Ebbinge BS (2002) Dark-Bellied Brent Geese Branta bernicla bernicla, as recorded by satellite telemetry, do not minimize flight distance during spring migration. Ibis 144:106-121

Guo-Gang Z, Dong-Ping L, Yun-Qiu H, Hong-Xing J, Ming D, Fa-Wen Q, Jun L, Zhi X, Feng-Shan L (2011) Migration routes and stop-over sites determined with satellite tracking of bar-headed geese Anser indicus breeding at Qinghai Lake, China. Waterbirds 34:112-116

Hawkes LA, Balachandran S, Batbayar N, Butler PJ, Chua B, Douglas DC, Frappell PB, Hou Y, Milsom WK, Newman SH, Prosser DJ, Sathiyaselvam P, Scott GR, Takekawa JY, Natsagdorj T, Wikelski M, Witt MJ, Yan B, Bishop CM (2011) The trans-Himalayan flights of bar-headed geese (Anser indicus). PNAS 108:9516-9519

Hawkes LA, Balachandran S, Batbayar N, Butler PJ, Frappell PB, Milsom WK, Tseveenmyadag N, Newman SH, Scott GR, Sathiyaselvam P, Takekawa JY, Wikelski M, Bishop CM (2013) The paradox of extreme high-altitude migration in bar-headed geese Anser indicus. Proc R Soc B 280:20122114

Hussey NE, Kessel ST, Aarestrup K, Cooke SJ, Cowley PD, Fisk AT, Harcourt RG, Holland KN, Iverson SJ, Kocik JF, Flemming JEM, Whoriskey FG (2015) Aquatic animal telemetry: a panoramic window into the underwater world. Science 348:1255642

Kalra M, Kumar S, Rahmani AR, Khan JA, Mohammed Belal S, Masood Khan A (2011) Satellite tracking of bar-headed Geese Anser indicus wintering in Uttar Pradesh, India. J Bombay Nat Hist Soc 108:79

Kays R, Crofoot MC, Jetz W, Wikelski M (2015) Terrestrial animal tracking as an eye on life and planet. Science 348:aaa2478 
Keeley ATH, Beier P, Keeley BW, Fagan ME (2017) Habitat suitability is a poor proxy for landscape connectivity during dispersal and mating movements. Landsc Urban Plan 161:90-102

LaPoint S, Gallery P, Wikelski M, Kays R (2013) Animal behavior, cost-based corridor models, and real corridors. Landscape Ecol 28:1615-1630

McRae BH, Dickson BG, Keitt TH, Shah VB (2008) Using circuit theory to model connectivity in ecology, evolution, and conservation. Ecology 89:2712-2724

Michelot T, Langrock R, Bestley S, Jonsen ID, Photopoulou T, Patterson TA (2017) Estimation and simulation of foraging trips in land-based marine predators. Ecology 98:1932-1944

Morales JM, Haydon DT, Frair J, Holsinger KE, Fryxell JM (2004) Extracting more out of relocation data: building movement models as mixtures of random walks. Ecology $85: 2436-2445$

Nathan R, Getz WM, Revilla E, Holyoak M, Kadmon R, Saltz D, Smouse PE (2008) A movement ecology paradigm for unifying organismal movement research. PNAS 105:19052-19059

Newman SH, Hill NJ, Spragens KA, Janies D, Voronkin IO, Prosser DJ, Yan B, Lei F, Batbayar N, Natsagdorj T, Bishop CM, Butler PJ, Wikelski M, Balachandran S, Mundkur T, Douglas DC, Takekawa JY (2012) Eco-virological approach for assessing the role of wild birds in the spread of avian influenza H5N1 along the Central Asian Flyway. PLoS ONE 7:e30636

Patterson TA, Thomas L, Wilcox C, Ovaskainen O, Matthiopoulos J (2008) State-space models of individual animal movement. Trends Ecol Evol 23:87-94

Péron G, Fleming CH, de Paula RC, Mitchell N, Strohbach M, Leimgruber P, Calabrese JM (2017) Periodic continuoustime movement models uncover behavioral changes of wild canids along anthropization gradients. Ecol Monogr 87:442-456

Prosser DJ, Cui P, Takekawa JY, Tang M, Hou Y, Collins BM, Yan B, Hill NJ, Li T, Li Y, Lei F, Guo S, Xing Z, He Y, Zhou Y, Douglas DC, Perry WM, Newman SH (2011) Wild bird migration across the Qinghai-Tibetan plateau: a transmission route for highly pathogenic H5N1. PLoS ONE 6:e17622

Richard Y, Armstrong DP (2010) Cost distance modelling of landscape connectivity and gap-crossing ability using radio-tracking data. J Appl Ecol 47:603-610
Row JR, Blouin-Demers G, Lougheed SC (2010) Habitat distribution influences dispersal and fine-scale genetic population structure of eastern foxsnakes (Mintonius gloydi) across a fragmented landscape. Mol Ecol 19:5157-5171

Squires JR, DeCesare NJ, Olson LE, Kolbe JA, Hebblewhite M, Parks SA (2013) Combining resource selection and movement behavior to predict corridors for Canada lynx at their southern range periphery. Biol Conserv 157:187-195

Takekawa JY, Heath SR, Douglas DC, Perry WM, Javed S, Newman SH, Suwal RN, Rahmani AR, Choudhury BC, Prosser DJ, Yan B, Hou Y, Batbayar N, Natsagdorj T, Bishop CM, Butler PJ, Frappell PB, Milsom WK, Scott GR, Hawkes LA, Wikelski M (2009) Geographic variation in bar-headed geese Anser indicus : connectivity of wintering areas and breeding grounds across a broad front. Wildfowl 59:102-125

Technitis G, Weibel R, Kranstauber B, Safi K (2016) An algorithm for empirically informed random trajectory generation between two endpoints. In: GIScience 2016: Ninth International Conference on Geographic Information Science

Technitis G, Weibel R, Kranstauber B, Safi K (in preparation) On old tracks to new insight: random trajectories on recorded paths

Thurfjell H, Ciuti S, Boyce MS (2014) Applications of stepselection functions in ecology and conservation. Mov Ecol $2: 4$

Turner GG, Reeder D, Coleman JT (2011) A five-year assessment of mortality and geographic spread of white-nose syndrome in North American bats, with a look at the future. Update of white-nose syndrome in bats. Bat Res News 52:13-27

van Toor ML, Newman SH, Takekawa JY, Wegmann M, Safi K (2016) Temporal segmentation of animal trajectories informed by habitat use. Ecosphere 7:e01498

Zeigler SL, Fagan WF (2014) Transient windows for connectivity in a changing world. Mov Ecol 2:1

Zeller KA, McGarigal K, Cushman SA, Beier P, Vickers TW, Boyce WM (2014) Sensitivity of landscape resistance estimates based on point selection functions to scale and behavioral state: pumas as a case study. Landscape Ecol 29:541-557

Zeller KA, McGarigal K, Cushman SA, Beier P, Vickers TW, Boyce WM (2016) Using step and path selection functions for estimating resistance to movement: pumas as a case study. Landscape Ecol 31:1319-1335 